\title{
О ВОЗМОЖНОСТИ РАСПРОСТРАНЕНИЯ ОДНОМЕРНОИ АКУСТИЧЕСКОЙ ВОЛНЫ СТАЦИОНАРНОГО ПРОФИЛЯ В НЕЛИНЕИНОМ СТАНДАРТНОМ ВЯЗКОУПРУГОМ ТЕЛЕ
}

A. STULOV. STATSIONAARSE PROFIILIGA OHEMOOTMELISE AKUSTILISE LAINE LEVI VOIMALIKKUSEST MITTELINEAARSES STANDARDSES VISKOELASTSES KESKKONNAS

A. STULOV. ON THE ONE-DIMENSIONAL ACOUSTIC WAVE OF A CONSTANT PROFILE PROPAGATION IN NONLINEAR STANDARD VISCOELASTIC MEDIUM

\section{(Представил Н. Алумяэ)}

Существование волн, распространяющихся в нелинейных средах без изменения формы и скорости, известно давно, и они достаточно подробно описаны, например, в монографиях $\left[{ }^{1,2}\right]$. Такие же волны в нелинейных наследственных средах изучены меньше. В $\left[{ }^{3}\right]$ показана возможность существования в нелинейной наследственной среде волн стационарного профиля, движущихся со скоростью, равной скорости звука в линейной среде, а в $\left[{ }^{4}\right]$, движущихся с большей скоростью.

Нами исследуются условия существования и форма волн стационарного профиля, скорость которых зависит от амплитуды.

Рассмотрим одномерный нелинейный волновой процесс, зависящий от лангранжевой координаты $X$, времени $t$, описываемый уравнением

$$
U^{*}(X, t)-c^{2} g\left(U^{\prime}\right) U^{\prime \prime}(X, t)+\int_{0}^{t} K(\tau) U^{*}(X, t-\tau) d \tau=0,
$$

и краевую задачу с условием

$$
U \cdot(0, t)=-c A H(t) \varphi(t), \quad A=\text { const, }
$$

где $U(X, t)$ - продольные перемещения, $g\left(U^{\prime}\right)$ - функция нелинейности среды, $K(\tau)$ - ядро ползучести наследственной среды, $c$ - скорость звука в среде, $H(t)$ - функция Хевисайда, $\varphi(t)$ - гладкая функция. Штрих обозначает производную по $X$, а точка - производную по $t$.

Выберем нулевые начальные условия

$$
U(X, 0)=0, \quad U \cdot(X, 0)=0,
$$

и функцию нелинейности в виде

$$
g\left(U^{\prime}\right)=1+\alpha U^{\prime}(X, t), \quad \alpha=\text { const. }
$$

Решение задачи (1), (2) будем искать в виде

$$
U(X, t)=H(\xi) \psi(\xi), \quad \xi=\frac{1}{2 \tau_{0}}\left(t-\frac{X}{V}\right),
$$

т. е. волны, вызванной краевым воздействием в точке $X=0$ и распространяющейся со скоростью $V$ в положительном направлении оси $X$. 
Введенный в формулу (5) параметр $\tau_{0}$ имеет размерность времени и будет определен ниже.

Функция $\psi(\xi)$ удовлетворяет условиям

$$
\left|\psi^{\cdot}(\xi)\right|<\infty, \quad \psi(0)=0, \quad \psi^{\cdot}(0)=B=\text { const. }
$$

\section{Обозначим}

$$
1-\frac{c^{2}}{V^{2}}=a_{\varepsilon}, \quad \beta=\frac{\alpha c^{2}}{2 \tau_{0} \varepsilon V^{3}},
$$

и выберем $B=-a / \beta$. Тогда, подставляя (5) в (1), для $\xi>0$ получим уравнение

$a \varepsilon \psi^{\prime \prime}(\xi)+\beta \varepsilon \psi^{\prime}(\xi) \psi^{\prime \prime}(\xi)+2 \tau_{0} B K\left(2 \tau_{0} \xi\right)+2 \tau_{0} \int_{0}^{\xi} K\left[2 \tau_{0}(\xi-z)\right] \psi^{\prime \prime}(z) d z=0$.

Рассмотрим случай стандартного вязкоупругого тела, для которого

$$
K(t-\tau)=\left(\varepsilon / \tau_{0}\right) \exp \left(-\frac{t-\tau}{\tau_{0}}\right),
$$

где $\varepsilon>0$ и $\tau_{0}>0-$ наследственные параметры среды. Обозначив $\beta \psi^{\cdot}(\xi)=W(\xi)$, получим уравнение

$$
W \cdot(\xi) e^{2 \xi}[a+W(\xi)]-2 a+2 \int_{0}^{\xi} e^{2 z} W \cdot(z) d z=0 .
$$

Дифференцируя уравнение (10) по $\xi$, а затем дважды интегрируя и учитывая, что $\mathbb{W}(0)=-a$, найдем

$$
\left[\frac{W(\xi)+W_{1}}{W_{1}-a}\right]^{p}\left[\frac{W(\xi)+W_{2}}{W_{2}-a}\right]^{1-p}=\exp (-\xi),
$$

где

$$
\begin{gathered}
W_{1}=1+a+C_{1}, \quad W_{2}=1+a-C_{1}, \\
p=\left(W_{1}-a\right) /\left(W_{1}-W_{2}\right) .
\end{gathered}
$$

Константу интегрирования $C_{1}$ необходимо определить, подставив полученное решение в уравнение (10). Однако, сделать это затруднительно, так как решение получено в неявном виде. Выбрав $p=1$ или $p=0$, получим

$$
W(\xi)=2\left(e^{-\xi}-1\right)-a .
$$

Это решение удовлетворяет уравнению (10) только при $a=0$, т. е. для $V=c$. Этот результат был получен в $\left.{ }^{3}\right]$.

Положив $p=2$ или $p=-1$, можно найти решения уравнения (10)

$$
W(\xi)=\frac{4}{3} e^{-\xi}\left[1-\frac{2}{3} e^{\xi}+\sqrt{1-e^{\ddagger}}\right],
$$

при условии $a=-\frac{4}{9}$. Это решение не является подходящим, так как оно комплексное при $\xi>0$.

Введем новую переменную $y(\xi)$, так что

$$
W(\xi)=\frac{2(\gamma-1)}{(\gamma-2)}[y(\xi)-1]-a, \quad \gamma=p^{-1} .
$$

Для того, чтобы было выполнено условие $W(0)=-a$, необходимо выполнение условия

$$
y(0)=1
$$


Из (11) найдем, что̄ $y(\xi)$ удовлетворяет алгебра́ическому уравнению

$$
[\gamma-(\gamma-1) y(\xi)][y(\xi)]^{\gamma-1}=\exp (-\gamma \xi)
$$

Это уравнение имеет два различных вещественных решения $y_{1}(\xi)$ и $y_{2}(\xi)$, удовлетворяющих условию (16) при любых $\xi>0$ и для всех $\gamma>1(\gamma \neq 2)$. Если $\gamma=2$, то ограниченное решение (8) существует только при $\varepsilon=0$.

Используя (15) - (17), найдем, что уравнение (10) удовлетворяется только при

$$
1-\frac{c^{2}}{V^{2}}=a \varepsilon=\frac{2 \varepsilon(\gamma-1)}{(\gamma-2)^{2}}, \quad \gamma>1, \quad \gamma \neq 2
$$

Так как $a>0$ для всех $\gamma>1$, и при

$$
\varepsilon<\frac{(\gamma-2)^{2}}{2(\gamma-1)}, \quad \varepsilon a<1,
$$

To

$$
V=c(1-a \varepsilon)^{-1 / 2}>c .
$$

Таким образом, в нелинейном стандартном вязкоупругом теле могут распространяться с одной и той же скоростью $V>c$ две различные по форме волны стационарного профиля

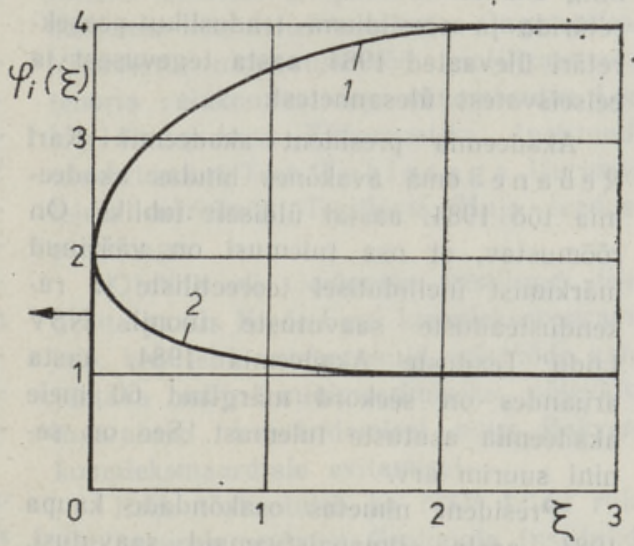

Профили импульсов.

$$
U_{i}(X, t)=\left(2 \tau_{0} \beta\right)^{-1} H(\xi) W(\xi), \quad i=1,2,
$$

возбуждаемые краевым условием (2), с амплитудой

$$
A=\frac{2 \varepsilon(\gamma-1)}{\alpha(\gamma-2)} \frac{V^{3}}{c^{3}},
$$

и функцией $\varphi(t)$, определяющей форму волны, задаваемой в виде

$$
\varphi_{i}(\xi)=\frac{\gamma-1}{\gamma-2}-y_{i}(\xi), \quad i=1,2,
$$

где $y_{i}(\xi)$ вещественные решения уравнения (17), удовлетворяющие условию (16), а $V$ определено в (20).

На рисунке показаны два профиля импульсов $\varphi_{i}(\xi)$ распространяющихся со скоростью $V>c$ в положительном направлении оси $X$ без искажения формы для случая $\gamma=3$.

\section{Л ИТЕРА Т Р А}

1. Уизем Дж. Линейные и нелинейные волны. М., «Мир», 1977.

¿. Карпман В. Н. Нелинейные волны в диспергирующих средах. М., «Наука», 1973.

3. Nigul, $U$. In: Nonlinear Deformation Waves, IUTAM Symp., Tallinn, 1982, Berlin, Springer, 1983.

4. Стулов A. C. Тез. докл. IV Всесоюз. симпозиума по физике акусто-гидродинамических явлений и оптоакустике с секциями молекулярной акустики и геоакустики. Ашхабад, 1985, 31 .

Ннститут кибернетики Академии наук Эстонской ССР
Поступила в редакцию 23/XII 1985 